\author{
Francesco Ferrara \\ (dottorando di ricerca in "Diritto e Tutela", nell'Università degli Studi di Roma \\ "Tor Vergata", Dipartimento di Giurisprudenza)
}

\title{
Lex orandi, lex credendi? \\ Il Messale Romano e il suo valore simbolico tra Summorum Pontificum e Traditionis Custodes *
}

Lex orandi, lex credendi?

The Roman Missal and its symbolic value between Summorum Pontificum and

Traditionis Custodes *

\begin{abstract}
This essay aims to examine the symbolic value acquired by the Roman Missal of 1962 by the Second Vatican Council today, especially through the comparison of two recent statutes, Summorum Pontificum and Traditionis Custodes. They can represent an effective synthesis of the approaches used by the Catholic Church to relate to a liturgical question that is still very controversial.
\end{abstract}

SOMMARIO: 1. Il "pomo della discordia": Concilio Vaticano II - 2. L'ermeneutica della riforma nella continuità - 3. Unità nella unicità? - 4. Esempi di non trascurabile fedeltà.

\section{1 - Il "pomo della discordia": Concilio Vaticano II}

Il ventunesimo Concilio ecumenico, Vaticano II (11 ottobre 1962 - 8 dicembre 1965), ha rappresentato un momento spartiacque per la costruzione del complesso rapporto della Chiesa cattolica con la modernità; significativamente, esso faceva seguito a un Concilio - Vaticano I (8 dicembre 1869 - 20 ottobre 1870) - sospeso sine die a causa dell'irrompere della Storia, guerra franco-prussiana e occupazione di Roma, entro i Sacri Palazzi ${ }^{1}$.

\footnotetext{
* Il contributo, sottoposto a valutazione, riproduce il testo dell'intervento presentato al webinar "I simboli religiosi nella società contemporanea" (Milano 23-24 settembre 2021), i cui Atti sono in corso di pubblicazione presso la casa editrice Giappichelli.

${ }^{1}$ Per un'ampia disamina vedi L.G. DEHON, Diario del Concilio Vaticano I, a cura di V. CARBONE, Tipografia Poliglotta Vaticana, Città del Vaticano, 1962.
} 
L'evento che poneva fine al millenario potere temporale dei papi si innestava, però, in un processo di più vasta portata, tale da imporre anche una riflessione sul ruolo da attribuirsi al potere spirituale in una società che andava rapidamente secolarizzandosi. Cresceva nella Chiesa il movimento "modernista", così inizialmente appellato solo dai suoi detrattori, che piuttosto si definiva come gruppo di cattolici "viventi in armonia con lo spirito del loro tempo" 2 ; dunque, a questo fine, desiderosi di adeguare il cattolicesimo alle sollecitazioni del Secolo.

Questa corrente di pensiero trovò in Pio $\mathrm{X}$ il suo più fiero avversario, infatti, con l'enciclica Pascendi Dominici Gregis (8 settembre 1907), papa Sarto intraprese rigide misure in chiave antimodernista giungendo, addirittura, a richiedere un apposito giuramento al clero con il Motu Proprio Sacrorum Antistitum (1을 settembre 1910) ${ }^{3}$.

La dialettica tra istanze riformiste e difesa della tradizione continuò, comunque, con alterne vicende, a caratterizzare il cammino della Chiesa nel Novecento; segno di una comune esigenza volta a definire il ruolo del cattolicesimo, in un mondo che usciva dalle due guerre mondiali e affrontava grandi sfide sociali e ideologiche. Il desiderio di un Concilio ecumenico raggiunse un'effettiva possibilità di realizzazione già con Pio XII, furono anche compiuti concreti passi ${ }^{4}$ in vista dell'obiettivo, ma non se ne fece nulla sino al papato dell'ottuagenario Giovanni XXIII, che, a soli tre mesi dall'elezione al soglio pontificio, manifestò ufficialmente questo proponimento, il 25 gennaio 1959, dalla Sala capitolare del Monastero di San Paolo 5 .

Il Concilio che si chiuse nel 1965, durante il papato di Paolo VI, rivolgeva così "la mente della Chiesa $[\ldots]$ verso la direzione

2 Il programma dei modernisti. Risposta all'enciclica di Pio X "Pascendi Dominici Gregis", Fratelli Bocca, Torino, 1911, p. 7. L'autore di questo manifesto del modernismo resta ancora anonimo, la prefazione della traduzione francese è a firma di Guglielmo Quadrotta, giovane laico modernista e direttore (1909-1914) della rivista Cultura contemporanea. Così: A. BEA, L'Enciclica «Pascendi» e gli studi biblici: nel $50^{\circ}$ anniversario dell'importante documento, in Biblica, II, 1958, p. 127 (in nota).

${ }^{3}$ Sulle conseguenze dell'applicazione di Pascendi Dominici Gregis vedi AA. VV., The Reception and Application of the Encyclical Pascendi. The Reports of the Diocesan Bishops and the Superiors of the Religious Orders until 1914, a cura di C. ARNOLD, G. VIAN, Edizioni Ca' Foscari, Venezia, 2017.

${ }^{4}$ G. CAPRILE (a cura di), Il Concilio Vaticano II. Annunzio e preparazione, vol. I, parte I, 1969-1960, La Civiltà Cattolica, Roma, 1966, p. 15.

${ }^{5}$ Acta Apostolicae Sedis, Allocuzione del Santo Padre Giovanni XXIII con la quale annuncia il Sinodo Romano, il Concilio Ecumenico e l'aggiornamento del Codice di diritto canonico, 25 gennaio 1959, vol. LI, 1959, pp. 65-69. 
antropocentrica della cultura moderna» ${ }^{6}$, ponendosi quale fondamentale elemento entro cui si auto-riconosce il cattolicesimo; dunque, eventosignificante non solo sul piano endo-religioso, ma anche dal punto di vista di una "storiografia di impianto decisamente più secolare"7.

Questa apertura del Concilio alla modernità ha prodotto una serie di documenti innovativi rispetto al tradizionale Magistero della Chiesa, si pensi soprattutto all'ecumenismo (decreto Unitatis Redintegratio), al dialogo interreligioso - soprattutto avuto riguardo ai rapporti con l'ebraismo - (dichiarazione Nostra Aetate), al principio della libertà religiosa (dichiarazione Dignitatis Humanae) e al ruolo della Chiesa nel mondo moderno (costituzione Gaudium et Spes) ${ }^{8}$.

Un tale radicale rinnovamento non poteva trovare unanimi consensi, infatti, dai lavori conciliari sino ai nostri giorni, diverse sono state le posizioni critiche o di aperto dissenso su talune di queste determinazioni e la loro ermeneutica; in proposito, basti solo pensare al ruolo tuttora ricoperto dalla Fraternità Sacerdotale San Pio X (FSSPX), fondata da Mons. Marcel Lefebvre nel 1970. Oggetto precipuo di questa trattazione, però, è una questione liturgica, entro cui sembrano trovare corrispondenza tutti i movimenti "tradizionalisti"9.

Si tratta dell'opposizione al Novus Ordo Missae del 1969 di Paolo VI, promulgato in attuazione ${ }^{10}$ dei principi stabiliti dal Vaticano II; una "revisione" del Messale Romano - lex orandi della Chiesa cattolica, pur con alcune modifiche, almeno dal Concilio di Trento (1570) - che, soprattutto, investiva le preghiere eucaristiche, l'orientamento del celebrante durante la Messa, la musica sacra e l'uso del latino (divenuto rapidamente marginale a vantaggio delle lingue nazionali).

6 Allocuzione del Santo Padre Paolo VI, ultima sessione pubblica del Concilio ecumenico Vaticano II, 7 dicembre 1965, in www.vatican.va.

7 F. TRANIELlO (a cura di), Il concilio Vaticano II nella storia del Novecento, in Il Vaticano II nella storia del Novecento. Interventi a cura di Francesco Traniello, in Contemporanea, III, 2003, p. 521 .

8 Per approfondire la sterminata bibliografia sul Concilio Vaticano II si rinvia a: G. ALBERIGO (diretta da), Storia del Concilio Vaticano II, a cura di A. MELLONI, il Mulino, Bologna, 1995-2001, 5 voll.; E. GALAVOTTI, Una bibliografia sul concilio Vaticano II, in Atlante storico del Concilio Vaticano II, a cura di A. MeLlONI, Jaca Book, Milano, 2015.

${ }^{9}$ P. CHENAUX, Recensione a N. BUONASORTE, Tra Roma e Lefebvre. Il tradizionalismo cattolico italiano e il Concilio Vaticano II, con Prefazione di R. MOROZZO DELLA ROCCA, Edizioni Studium, Roma, 2003, in Rivista di storia della Chiesa in Italia, II, 2006, p. 585.

${ }^{10} \mathrm{Il}$ riferimento è soprattutto alla costituzione Sacrosanctum Concilium. 
Il Messale Romano preconciliare, promulgato da Giovanni XXIII nel 1962, assurse, così, a simbolo di un cristianesimo "di minoranza", che trasversalmente univa i tradizionalisti nel tentativo di mantenere integro l'antico rito, quale proprio essenziale elemento identitario, anche nel mondo contemporaneo. La difesa della "Messa in latino" non fu però appannaggio di uno sparuto gruppo di dissidenti, ma interessò personalità di primo piano anche all'interno della gerarchia ecclesiastica, basti pensare che il saggio «Breve esame critico del Novus Ordo Missae», redatto da un gruppo di teologi cattolici, venne presentato a Paolo VI con una lettera di presentazione dei cardinali Ottaviani e Bacci che non esitava a definire il nuovo rito "un impressionante allontanamento dalla teologia cattolica della Santa Messa"11.

Al di là delle questioni puramente liturgiche, l'antica lex orandi della Chiesa trovò argomenti a proprio favore anche oltre il campo fideistico; un esempio di questa "riscoperta" può essere fornito dal celebre Appeal to preserve Mass sent to Vatican pubblicato sul Times del 6 luglio 1971, a firma di un gruppo di intellettuali inglesi, anche lontani dal cristianesimo. La Messa cattolica romana andava preservata quale «tradizione vivente», ispiratrice di capolavori artistici in tutte le epoche e patrimonio della cultura universale non meno che della Chiesa e dei suoi fedeli. Questo appello consentì la marginale sopravvivenza del Vetus Ordo in Inghilterra, in virtù di un indulto concesso da Paolo VI il 30 ottobre del 1971, ricordato come "indulto di Agatha Christie", la celebre scrittrice era, infatti, tra i firmatari ${ }^{12}$.

Il Messale Romano preconciliare continuò, comunque, a essere utilizzato dai vari gruppi tradizionalisti, che trovarono nell'opera di Mons. Lefebvre il proprio principale riferimento, anche in virtù di un indirizzo ermeneutico post-conciliare segnato da profondi tratti di discontinuità rispetto al suo immediato, e millenario, passato.

All'esistenza di una contrapposta "legge di continuità", entro cui interpretare i documenti conciliari, faceva già riferimento l'allora prof. J. Ratzinger, all'annuale Convegno dei cattolici di lingua tedesca del 1966 a Bamberga ${ }^{13}$.

11 AA. VV., Breve esame critico del «Novus Ordo Missae», a cura di INTER MultiPLICES UNA Vox, Presentato al Pontefice Paolo VI dai Cardinali A. Ottaviani e A. Bacci nel settembre del 1969 (in www.unavox.it).

12 Per approfondire vedi G. AMATO, L'indulto di Agatha Christie. Come si è salvata la Messa tridentina in Inghilterra, Fede \& Cultura, Verona, 2013.

13 J. RATZINGER, Il cattolicesimo dopo il Concilio, Conferenza al Katholikentag di Bamberga del 1966, in ID., Il nuovo popolo di Dio. Questioni ecclesiologiche, Editrice 


\title{
2 - L'ermeneutica della riforma nella continuità
}

La celebrazione secondo il Vetus Ordo costituiva l'elemento aggregante di una compagine tradizionalista piuttosto eterogena, entro cui potevano rintracciarsi sia movimenti attenti alla sola questione liturgica, sia visioni in aperta opposizione con il cammino tracciato dal Vaticano II. Il Messale Romano del 1962, significante del rito antico, andava così acquisendo un significato che, per alcuni, oltrepassava la sola difesa della "Messa in latino" e i tentativi di ricomposizione della frattura con la FSSPX, principale interlocutore sino a quel momento, fallirono sia con Paolo VI, sia con Giovanni Paolo II.

Soprattutto sotto il papato di Wojtyla sembrò vicina una soluzione che restituisse la piena comunione, $\mathrm{fu}$ anche firmato un Protocollo tra Mons. Lefebvre e il Card. Ratzinger (intanto divenuto prefetto della "Congregazione per la Dottrina della Fede") il 5 maggio 1988; ma, l'accordo fu poi denunciato da Mons. Lefebvre che, il 30 giugno dello stesso anno, ordinò quattro nuovi vescovi nonostante il formale monitum (17 giugno) inviato da Roma. La reazione non tardò ad arrivare, con il Motu Proprio Ecclesia Dei (2 luglio 1988), Giovanni Paolo II qualificò tale atto come «scismatico» e ammonì

\begin{abstract}
"tutti coloro che finora sono stati in diversi modi legati al movimento dell'Arcivescovo Lefebvre, affinché compiano il grave dovere di rimanere uniti al Vicario di Cristo nell'unità della Chiesa Cattolica, e di non continuare a sostenere in alcun modo quel movimento. Nessuno deve ignorare che l'adesione formale allo scisma costituisce una grave offesa a Dio e comporta la scomunica stabilita dal diritto della Chiesa"14.
\end{abstract}

Al contempo, a dimostrazione di una profonda conoscenza dei diversi orientamenti in seno al tradizionalismo, veniva concesso a coloro che si sentivano legati al Vetus Ordo la possibilità di continuare a utilizzare il Messale Romano del 1962, esortando i Vescovi a usare largamente tale facoltà, già prevista dall'indulto Quattuor abhinc annos (1984), in favore dei fedeli che lo avessero richiesto; ancora, veniva istituita la "Commissione Ecclesia Dei" con lo scopo di facilitare la piena comunione di coloro volevano restare uniti al Papa, "conservando le loro tradizioni spirituali e liturgiche", in applicazione del rammentato Protocollo. Questo

Queriniana, Brescia, 1992, 330 ss.

14 Lettera Apostolica Ecclesia Dei del Sommo Pontefice Giovanni Paolo II in forma di Motu Proprio, in www.vatican.va. 
provvedimento consentiva una prima separazione delle questioni liturgiche da quelle dottrinali, infatti, il "prezzo" della comunione con Roma era la sostanziale accettazione del Concilio Vaticano II, sia pure con un tollerato atteggiamento di critica positiva a certi suoi punti non fondamentali o con riferimento a riforme posteriori della liturgia e del diritto.

Il Messale Romano del 1962 restava il simbolo dei tradizionalisti, ma il perimetro dei suoi significati era ora visibilmente percorso dalle scelte degli aderenti a questa complessa galassia, verso cui non aveva perso d'interesse il Card. Ratzinger quando, nel 2005, giunse al soglio pontificio. Il teologo bavarese ebbe modo di chiarire alcune linee programmatiche del proprio pontificato già nel discorso di Natale alla Curia romana del 2005, in cui si soffermò sui problemi di ricezione incontrati dal Vaticano II; la critica era soprattutto rivolta a quella che viene definita "ermeneutica della discontinuità e della rottura", secondo cui i testi del Concilio ne rappresenterebbero in maniera imperfetta lo spirito, in quanto frutto di resistenze e compromessi.

"In una parola: occorrerebbe seguire non i testi del Concilio, ma il suo spirito"; esso sarebbe negli "slanci verso il nuovo che sono sottesi ai testi". Questa ermeneutica, continuando a seguire l'argomentare di Benedetto XVI, "non di rado si è potuta avvalere della simpatia dei massmedia, e anche di una parte della teologia moderna". A questa, però, si contrappone "l'ermeneutica della riforma", volta a rinnovare "nella continuità l'unico soggetto Chiesa"; i pur presenti elementi di discontinuità del Concilio non vanno, dunque, a imputarsi ai principi, ma alla ridefinizione dei rapporti della Chiesa con la scienza, lo Stato moderno e, più in generale, al tema della tolleranza religiosa. I due poli della continuità e della discontinuità convivono, così, su piani diversi; in questo equilibrio consisterebbe la "natura della vera riforma"15.

Nel tentativo di trovare una sintesi tra queste, in apparenza, inconciliabili tesi, Ratzinger si poneva l'obiettivo evitare una rottura definitiva tra "Chiesa preconciliare e postconciliare"; si trattava, così, anche della ricerca di una soluzione per i tradizionalisti, profondamente legati alla propria lex orandi, l'ermeneutica della continuità doveva poterne recuperare definitivamente il significato, oltre il simbolo, affinché il Messale preconciliare potesse tornare a essere un rito condiviso all'interno della Chiesa cattolica romana. Il Motu Proprio Summorum Pontificum del 7

15 Discorso di Sua Santità alla Curia Romana in occasione della presentazione degli auguri natalizi, 22 dicembre 2005 (in www.vatican.va). 
luglio 2007 esamina ex professo la questione, affermando il principio per cui il Messale promulgato da Paolo VI è espressione ordinaria della lex orandi della Chiesa, però, il Messale antico costituisce espressione straordinaria della stessa "legge della preghiera"; dunque, quali usi dello stesso rito romano, essi non possono portare "in alcun modo a una divisione nella lex credendi ("legge della fede") della Chiesa"16.

L'insegnamento di Benedetto XVI appare coerente con il Magistero dei suoi predecessori, trattandosi della stessa fede cattolica essa non potrebbe trovare, nei due usi, contraddizione nella lex orandi17. La "liberalizzazione" del Messale Romano del 1962 e la definitiva sistemazione canonica degli Istituti di vita consacrata e delle Società di vita apostolica che celebrano - spesso, abitualmente o permanentemente secondo il Vetus Ordo condusse, così, a una generale distensione con il fronte tradizionalista sia rispetto alle comunità che fin dal principio erano rimaste in piena comunione con Roma, sia avuto riguardo a coloro auspicavano rientrarvi con il riconoscimento di determinate garanzie liturgiche.

La via tracciata da Ratzinger non era però, come anticipato, nell'esclusivo solco della continuità, il Vaticano II restava irrinunciabile nel suo nucleo essenziale e a questa accettazione di discontinuità era pur sempre subordinato il ritorno, come dimostrano il provvedimento del 21 gennaio 2009 e la sua interpretazione del 10 marzo 200918. Il valore simbolico della "Messa in latino" tornava nel patrimonio immateriale della Chiesa postconciliare, consentendo, a un tempo, di meglio chiarire le posizioni dottrinali di molte compagini inserite nella galassia tradizionalista; esse, assai meno di prima, potevano assestare le proprie obiezioni intorno alla sola questione liturgica. L'ordito complesso tessuto dal papa teologo aveva individuato la "via stretta" attraverso cui porre le premesse per la coesistenza di sensibilità affatto diverse all'interno del cattolicesimo, un equilibrio ermeneutico potenzialmente duraturo, ma

16 Lettera Apostolica di Sua Santità Benedetto XVI "Motu Proprio data" Summorum Pontificum, 7 luglio 2007 (in www.vatican.va).

17 In particolare: «[...] se vogliamo distinguere e determinare in modo generale e assoluto le relazioni che intercorrono tra fede e Liturgia, si può affermare con ragione che "la legge della fede deve stabilire la legge della preghiera"». Così Pio XII nella Lettera Enciclica Mediator Dei «sulla Sacra Liturgia», 20 novembre 1947 (in www.vatican.va).

18 Cfr.: Decreto di remissione della scomunica latae sententiae ai Vescovi della Fraternità Sacerdotale San Pio X, 21 gennaio 2009 (in www.vatican.va); Lettera del Santo Padre Benedetto XVI ai Vescovi della Chiesa Cattolica riguardo alla remissione della scomunica del 4 Vescovi consacrati dall'Arcivescovo Lefebvre, 10 marzo 2009 (in www.vatican.va). 
fragile rispetto a orientamenti più restii a riconoscere la reciprocità insita nell'affermazione di un senso condiviso.

\section{3 - Unità nella unicità?}

La Declaratio di Benedetto XVI dell'11 febbraio 2013 ha rappresentato un evento eccezionale per la Chiesa cattolica, ossia la sua rinunzia al soglio di Pietro e l'elezione del Card. Bergoglio, il 13 marzo 2013, con il nome di Francesco.

I rapporti con i "tradizionalisti" del primo papa gesuita non sembrano potersi ricondurre a un unico schema rappresentativo; infatti, essi scontano un andamento ondivago su cui appare utile brevemente soffermarsi prima di provare a esaminarne gli esiti.

Il "Giubileo straordinario della Misericordia" del $2015 \mathrm{fu}$ introdotto da una lettera di indulgenza molto benevola, che prendeva anche in considerazione anche la FSSPX, prevedendo che la confessione amministrata dai suoi sacerdoti ai fedeli fosse pienamente valida e lecita per quell'anno, auspicando, altresì, di trovare presto soluzioni per il ristabilimento della piena comunione ${ }^{19}$; questa facoltà fu poi estesa oltre il periodo giubilare, "fino a nuove disposizioni in proposito"20. Addirittura, su proposta della "Congregazione per la Dottrina e la Fede" e della "Commissione Ecclesia Dei", Francesco approvò una lettera pontificia che disciplinava le modalità con cui sarebbe stato possibile ottenere una licenza per la celebrazione di matrimoni dei fedeli della FSSPX, "malgrado l'oggettiva persistenza "per ora" della situazione canonica di illegittimità"21.

Questi tentativi di riavvicinamento al principale soggetto tradizionalista, rimasto fuori dal Summorum Pontificum, fallirono definitivamente nel 2018, quando la distanza intorno ad alcune questioni dottrinali del Vaticano II, e del Magistero di Francesco, sembrò divenire insormontabile 22 .

19 Lettera del Santo Padre Francesco con al quale concede l'indulgenza in occasione del Giubileo straordinario della Misericordia, $1^{\circ}$ settembre 2015 (in www.vatican.va).

20 Lettera Apostolica Misericordia et misera del Santo Padre Francesco a conclusione del Giubileo straordinario della Misericordia, 20 novembre 2016 (in www.vatican.va).

${ }^{21}$ Lettera della Pontifica Commissione «Ecclesia Dei» ai Presuli delle Conferenze Episcopali interessate circa la licenza per la celebrazione di matrimonio di fedeli della Fraternità San Pio X, 27 marzo 2017 (in www.vatican.va).

22 Sul punto si rinvia all'intervista rilasciata dal nuovo superiore generale della FSSPX, 
Il Motu Proprio circa la Pontificia Commissione «Ecclesia Dei», del 19 gennaio 2019, torna così a volgere lo sguardo verso coloro che fin dal 1988 avevano deciso di restare in comunione con Roma e a quegli «Istituti e Comunità religiose che avevano aderito alla forma straordinaria del rito romano» in forza del Summorum Pontificum. La Commissione in argomento era quella preposta alla sorveglianza e all'applicazione delle disposizioni stabilite a vantaggio di quella parte del movimento tradizionalista che era restata, o era tornata, in piena comunione con la Chiesa cattolica; ebbene, essa venne soppressa e le sue competenze affidate a un'apposita sezione della "Congregazione per la Dottrina e la $F^{\prime \prime 2} e^{\prime 2}$, fondamentalmente sulla base dell'argomento per cui le questioni trattate nell'ambito del dialogo con la FSSPX fossero di carattere dottrinale, così come - prevalentemente - quelle della "Commissione Ecclesia Dei".

La decisione poteva essere interpretata sia come una razionalizzazione delle competenze, sia come parte di un processo più vasto, che ha poi portato, anche a seguito di ulteriori valutazioni, all'adozione del Motu Proprio Traditionis Custodes del 16 luglio 2021. Con questo provvedimento, introdotto da un'apposita lettera di presentazione ai Vescovi di tutto il mondo, Francesco abroga il Summorum Pontificum.

Un provvedimento che ha causato vasta eco nei movimenti tradizionalisti, con importanti riflessioni anche nel mondo dei media ${ }^{24}$, soprattutto ove essi godono di maggior seguito, come in Francia e negli USA; ma, Francesco, anche recentemente, ha avuto modo di ribadire come l'uso dei libri liturgici preconciliari si stesse trasformando in un'ideologia 25 .

Una supposta tendenza che, fin dall'art. 1, Traditionis Custodes, non fa mistero di voler minare alle fondamenta, affermando che i "libri

Davide Pagliarini, al Salzburger Nachrichten: J. BRUCKMOSER, Chef der Piusbrüder: "Dieser Papst erschüttert uns zutiefst", in Salzburger Nachrichten, 15 dicembre 2018, p. 11 ss.

${ }^{23}$ Cui era stata già più organicamente collegata da Benedetto XVI in forza del Motu Proprio Ecclesiae unitatem, 2 luglio 2009 (in www.vatican.va).

${ }^{24}$ Ex multis: J.M. GUENOIS, «Dieu seul le sait» $N^{\circ} 29$ - Messe en latin: pourquoi François est-il passé en force?, 25 luglio 2021, in www.lefigaro.fr; M.B. DOUGHERTY, Pope Francis Is Tearing the Catholic Church Apart, 12 agosto 2021 (in www.nytimes.com).

${ }^{25}$ Sul punto vedi l'intervista, in spagnolo, rilasciata a radio COPE: C. HERRERA, El Papa tras su operación: "Ni se me pasó por la cabeza renunciar", $1^{\circ}$ settembre 2021 (in www.vaticannews.va). 
liturgici promulgati [...] in conformità ai decreti del Concilio Vaticano II, sono l'unica espressione della lex orandi del Rito Romano"26.

Questo principio, così, sovverte l'interpretazione per cui Novus Ordo e Vetus Ordo possono considerarsi due "espressioni della lex orandi", come previsto dal Motu Proprio di Benedetto XVI; invece, il Messale postconciliare sembra divenire l'unico significante "costitutivo" 27 nella realtà del Rito Romano.

Nel suo significato letterale, inoltre, la pretesa unicità di questa lex orandi creerebbe conseguenze di non poco momento, se applicata in conformità al principio per cui "la legge della preghiera è legge della fede" (Lex orandi, lex credendi), poiché il Magistero della Chiesa insegna come la "Liturgia [...] non determina né costituisce il senso assoluto e per virtù propria la fede cattolica" 28 .

Anche accolta nel suo senso relativo, Traditionis Custodes ridetermina unilateralmente il significato dell'utilizzo del Messale Romano preconciliare, ergendolo a simbolo di un movimento apertamente contrario alla Chiesa postconciliare, come espressamente affermato nella lettera di presentazione ai Vescovi, infatti, risulterebbe

«sempre più evidente nelle parole e negli atteggiamenti di molti la stretta relazione tra la scelta delle celebrazioni secondo i libri liturgici precedenti al Concilio Vaticano II e il rifiuto della Chiesa e delle sue istituzioni in nome di quella che essi giudicano la "vera Chiesa"»29.

\section{4 - Esempi di non trascurabile fedeltà}

Si è fatto rapidamente cenno a quegli «Istituti di vita consacrata» e alle "Società di vita apostolica" che hanno largamente beneficiato del Summorum Pontificum, trovando in esso importanti garanzie liturgiche; questo, sia quelle in comunione con Roma fin dallo "strappo" di Mons.

26 Lettera Apostolica in forma di Motu Proprio del Sommo Pontefice Francesco Traditionis Custodes sull'uso della Liturgia Romana anteriore alla riforma del 1970, 16 luglio 2021 (in www.vatican.va).

27 Sul carattere "costitutivo" del significante nella realtà per J. Lacan vedi S. AUROUX, La filosofia del linguaggio, traduzione italiana di I. TANI, Editori Riuniti, Roma, 1998, p. 232.

28 Lettera Enciclica Mediator Dei «sulla Sacra Liturgia, cit.

29 Lettera del Santo Padre Francesco ai Vescovi di tutto il mondo per presentare il Motu Proprio «Traditionis Custodes» sull'uso della Liturgia Romana anteriore alla Riforma del 1970, 16 luglio 2021 (in www.vatican.va). 
Lefebvre del 1988, sia quelle di nuova costituzione o composte da soggetti desiderosi di rientrare entro uno stato canonico pienamente approvato dal soglio pontificio. Questi soggetti, in virtù del Protocollo LefebvreRatzinger del 1988, già promettono fedeltà alla Chiesa cattolica e dichiarano di accettare la dottrina del Concilio Vaticano II; riguardo certi punti controversi, essi si impegnano "ad assumere un atteggiamento positivo di studio e di comunicazione con la Sede Apostolica, evitando ogni polemica".

Il Summorum Pontificum permetteva ai parroci di accogliere le richieste di celebrazione secondo il Vetus Ordo da parte di gruppi stabili di fedeli, anche con licenza riguardo all'amministrazione dei sacramenti; ancora, se il parroco non avesse soddisfatto le richieste di questi gruppi, conformi al Motu Proprio, il Vescovo era "vivamente pregato di esaudire il loro desiderio". A più di un decennio dall'istaurazione di questa sorta di "pax liturgica", Traditionis Custodes segna una decisa linea di discontinuità.

Per i laici, si prevede l'esclusiva competenza del Vescovo a autorizzare l'uso del Messale Romano del 1962 (art. 2); dove esiste un gruppo stabile di fedeli che celebrano secondo il Vetus Ordo il Vescovo dovrà verificare la loro fedeltà alla riforma liturgica, ai dettati del Concilio Vaticano II e al Magistero dei Sommi Pontefici (art. 3, § 1), inoltre, nell'indicare a questi fedeli dove riunirsi per la celebrazione eucaristica, non potrà consentire l'utilizzo delle chiese parrocchiali (art. 3, § 2); infine, il Vescovo potrà valutare se mantenere o no le parrocchie personali canonicamente erette a beneficio di questi fedeli (art. $3, \S 5$ ).

Per i presbiteri, quelli ordinati dopo la pubblicazione di Traditionis Custodes potranno celebrare con il Messale del 1962 solo con autorizzazione del Vescovo, previa consultazione della Sede Apostolica (art. 4); invece, i presbiteri che già celebrano secondo il Messale del 1962 dovranno richiedere comunque al Vescovo un'autorizzazione per continuare ad avvalersi di tale facoltà (art. 5).

"Gli Istituti di vita consacrata e le Società di vita apostolica, a suo tempo eretti dalla Pontificia Commissione Ecclesia Dei passano sotto la competenza della Congregazione per gli Istituti di Vita Consacrata e le Società di Vita Apostolica" (art. 6);

sostanzialmente, perdendo un soggetto specificatamente dedicato alla loro supervisione. Da queste, pur minime, osservazioni non sembra potersi dubitare che la galassia dei movimenti tradizionalisti, in piena comunione con Roma, abbia subìto un mutamento di disciplina in peius rispetto a quanto previsto dal Summorum Pontificum; soprattutto cambia l'ermeneutica, il Messale preconciliare diviene il rinnovato simbolo di 


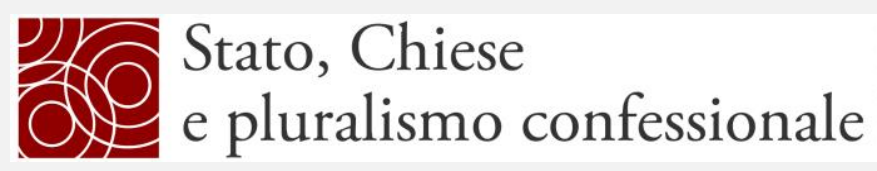

un'adesione mal tollerata, ridefinita in senso ideologico e lontana dalla contemporaneità.

Per coloro tra i tradizionalisti che hanno scelto la fedeltà al papato si profila, così, al di là dei meta-significati entro cui definire l'adesione al rito preconciliare, un futuro ancora da scrivere, nella difesa di una lex orandi divenuta, a un tempo, segno di distinzione e di appartenenza a un cattolicesimo che sembra spingerli verso un ruolo sempre più liminare. 